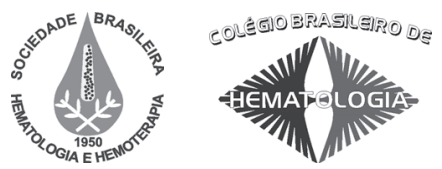

\title{
Cirurgias odontológicas em usuários de anticoagulantes orais
}

\section{Dental surgery in patients on oral anticoagulation therapy}

\author{
Alessandra K. Dantas ${ }^{I}$ \\ Maria Cristina Z. Deboni \\ José Luiz Piratininga ${ }^{3}$
}

\begin{abstract}
Em razão do crescimento do número de individuos submetidos à terapêutica anticoagulante também nos consultórios odontológicos, realizamos um levantamento retrospectivo de prontuários de pacientes anticoagulados com derivados cumarínicos e uma revisão sobre os protocolos de atendimento, a fim de procurar estabelecer diretrizes para um tratamento cirúrgico-odontológico adequado e seguro. A avaliação do paciente com relação ao seu nivel de anticoagulação através do Índice Normatizado Internacional (INR) ou Tempo de Protrombina (TP) e a classificação da amplitude do trauma cirúrgico são fatores importantes a serem avaliados antes do procedimento cirúrgico. Nosso levantamento mostrou que, em 47 cirurgias, sem alteração da medicação sistêmica, apenas um caso apresentou hemorragia pós-operatória, controlada por manobras de hemostasia local. Desse modo, observamos que, dentre os vários protocolos propostos na literatura, a manutenção da terapia anticoagulante, com a utilização de hemostáticos locais se necessário, parece o mais adequado à maioria dos casos cirúrgicos ambulatoriais. Rev. Bras. Hematol. Hemoter.
\end{abstract}

Palavras-chave: Anticoagulantes orais; terapia anticoagulante; hemostáticos locais; exodontia.

\section{Introdução}

Na prática clínica, as indicações de terapêutica anticoagulante vêm se tornando cada vez mais frequentes, consequentemente aumentando a ocorrência de pacientes usuários deste tipo de medicação nos consultórios odontológicos. Há uma grande diversidade de opiniões tanto na área médica quanto na odontológica em como realizar um tratamento odontológico seguro em pacientes anticoagulados com derivados cumarínicos. Vários protocolos de atendimento são sugeridos e incluem desde a interrupção completa do medicamento, sua redução ou a substituição pela heparina até a manutenção da terapia anticoagulante sem alteração, ${ }^{1-9}$ com ênfase na utilização de hemostáticos locais. ${ }^{10-19}$ Nenhum desses esquemas está livre de riscos, o que torna imprescindível uma avaliação completa da condição sistêmica do paciente, do acompanhamento do seu grau de anticoagulação através de exames laboratoriais e da classificação da amplitude do trauma cirúrgico envolvido no procedimento odontológico a ser realizado.

Esses protocolos vêm sendo pesquisados para garantir um tratamento que previna a ocorrência de sangramentos e, ao mesmo tempo, não exponha o paciente a um risco de tromboembolismo. Interromper a terapia anticoagulante, expondo dessa maneira o paciente a um risco desnecessário de tromboembolismo, não é uma atitude cautelosa, pois muitos autores demonstram que é seguro realizar a maioria dos procedimentos cirúrgicos odontológicos sem risco de hemorragias graves, quando o Índice Normatizado Internacional (INR) estiver dentro dos níveis terapêuticos.

Com objetivo de verificar o risco de sangramento pósoperatório em pacientes anticoagulados realizamos um levan-

${ }^{1}$ Cirurgiã-dentista.

${ }^{2}$ Cirurgiã-dentista. Professora Assistente do Departamento de Cirurgia da USP.

${ }^{3}$ Cirurgião-dentista. Professor Assistente do Depto. de Cirurgia da USP. Responsável pelo Serviço de Odontologia do Hospital Brigadeiro - UGA V.

Faculdade de Odontologia da Universidade de São Paulo - São Paulo-SP.

Correspondência: Alessandra K. Dantas

Av. Prof. Lineu Prestes, 2227 - Cidade Universitária

05508-900 - São Paulo-SP - Brasil

Tel.: (55 11) 9102-8116/(55 11) 3091-7832

E-mail: alekiyda@usp.br

Doi: 
tamento retrospectivo dos prontuários dos pacientes em uso de dicumarínicos, que foram atendidos no Hospital Brigadeiro, no período de 2003 a 2006, submetidos a cirurgias para avaliar a ocorrência desse tipo de complicação cirúrgica com e sem a utilização de hemostáticos locais.

\section{Casuística e Método}

Realizamos um levantamento retrospectivo de 26 prontuários de pacientes anticoagulados com dicumarínicos submetidos a cirurgias odontológicas ambulatoriais, atendidos no Serviço de Odontologia do Hospital Brigadeiro, no período de 2003 a 2006. Os dados anotados foram: idade na época do procedimento, gênero, valor de INR, tipo de cirurgia, a utilização ou não de algum hemostático local e a ocorrência de sangramento pós-operatório. Os pacientes foram operados sem realizar alterações na terapia anticoagulante. Todas as cirurgias foram realizadas pelo mesmo cirurgião, respeitando os princípios fundamentais da cirurgia. Qualquer complicação cirúrgica relacionada à hemorragia ou infecção foi considerada.

\section{Resultados}

Do total de 26 prontuários, os pacientes tinham idade entre 11 e 89 anos, sendo 18 homens e 8 mulheres. As cirurgias odontológicas a que foram submetidos corresponderam a 46 exodontias e uma apicectomia, de pequeno ou médio trauma cirúrgico (onde é esperado pouco ou mínimo sangramento trans e pós-operatório). Em 11 pacientes não foi utilizado nenhum tipo de hemostático; em 13 foi utilizado o Beriplast $\AA$ e em 2, o Tissucol® (Tabela 1).

Todos os pacientes operados estavam com INR (Índice Normatizado Internacional) dentro dos níveis terapêuticos para o tratamento médico que necessitavam (entre 1.8 e 3.6).

Não houve nenhum caso de sangramento pós-operatório nas cirurgias sem hemostático local. $\mathrm{O}$ único caso de hemorragia ocorreu na exodontia do dente 46 - molar inferior

Tabela 1. Tipo de cirurgia, valor do INR, uso de hemostático local e ocorrência de sangramento

\begin{tabular}{|c|c|c|c|c|c|}
\hline Cirurgia & Idade & Sexo & Inr & Adesivo & $\begin{array}{r}\text { Sangramento } \\
\text { pós-operatório }\end{array}$ \\
\hline Exodontia molar & 56 & $M$ & 2.1 & Beriplast & não \\
\hline Exodontia molar & 68 & $M$ & 3.2 & Beriplast & não \\
\hline Exodontia molar & 63 & M & 3.8 & Beriplast & $\operatorname{sim}$ \\
\hline Exodontia pré-molar & 65 & $\mathrm{~F}$ & 2.8 & Beriplast & não \\
\hline Exodontia $3^{\circ}$ molar incluso & 55 & $\mathrm{~F}$ & 3.0 & Beriplast & não \\
\hline Exodontia decíduo & 11 & $\mathrm{~F}$ & 1.9 & Beriplast & não \\
\hline Exodontia $3^{\circ}$ molar incluso & 55 & $M$ & 2.4 & Beriplast & não \\
\hline Exodontia pré-molar & 65 & $\mathrm{~F}$ & 2.6 & Beriplast & não \\
\hline Exodontia molar & 56 & M & 2.2 & Beriplast & não \\
\hline Exodontia molares (2) & 55 & $M$ & 3.1 & Tissucol & não \\
\hline Exodontia incisivos (2) & 50 & M & 2.5 & Tissucol & não \\
\hline Exodontia $3^{\circ}$ molar incluso & 39 & M & 2.9 & Beriplast & não \\
\hline Exodontia incisivo, canino e pré-molar & 39 & $\mathrm{~F}$ & 2.3 & Beriplast & não \\
\hline Exodontia $3^{\circ}$ molar incluso & 36 & M & 2.4 & Beriplast & não \\
\hline Exodontia pré-molar & 77 & $\mathrm{~F}$ & 2.2 & nenhum & não \\
\hline Exodontia incisivo (2) & 72 & M & 2.3 & nenhum & não \\
\hline Exodontia incisivo e canino & 68 & M & 3.1 & nenhum & não \\
\hline Exodontia molar & 73 & M & 2.2 & nenhum & não \\
\hline Exodontia pré-molares (2) & 57 & M & 2.8 & nenhum & não \\
\hline Exodontia molar & 58 & M & 3.5 & nenhum & não \\
\hline Exodontia molar (2) & 89 & M & 3.6 & nenhum & não \\
\hline Exodontia caninos (2) & 63 & M & 2.2 & nenhum & não \\
\hline Exodontia incisivo & 75 & $M$ & 2.7 & nenhum & não \\
\hline Exodontia pré-molar e molar & 41 & M & 2.0 & nenhum & não \\
\hline Exodontia molar & 41 & $M$ & 2.2 & nenhum & não \\
\hline Exodontia pré-molar e molar & 76 & $\mathrm{~F}$ & 2.1 & nenhum & não \\
\hline Exodontia molar (2) & 76 & $\mathrm{~F}$ & 2.3 & nenhum & não \\
\hline Exodontia incisivo e pré-molar & 76 & $\mathrm{~F}$ & 2.5 & nenhum & não \\
\hline Exodontia canino e pré-molar & 73 & M & 2.8 & nenhum & não \\
\hline Exodontia molar & 69 & $\mathrm{~F}$ & 1.9 & nenhum & não \\
\hline Apicectomia de canino & 69 & $\mathrm{~F}$ & 2.2 & nenhum & não \\
\hline Exodontia pré-molar & 75 & $\mathrm{~F}$ & 2.5 & nenhum & não \\
\hline Exodontia pré-molar & 71 & $M$ & 1.8 & nenhum & não \\
\hline
\end{tabular}


(INR de 3.8 e utilizado o Beriplast ${ }^{\circledR}$ ), dois dias após o procedimento cirúrgico, o qual foi controlado apenas com medidas locais de hemostasia realizando tamponamento com gaze. Não houve descrição de quadro de infecção em nenhum dos casos. A tabela 1 expressa os dados considerados.

\section{Revisão de literatura e discussão}

A utilização de anticoagulantes orais de uso contínuo por indivíduos com algum tipo de comprometimento vascular ou cardíaco torna imprescindível a realização de uma anamnese cuidadosa quanto ao controle de episódios hemorrágicos ou tromboembólicos, e de uma avaliação clínica multiprofissional.

O controle médico do grau de anticoagulação do paciente é imperativo e deve ser realizado periodicamente para verificar se o nível terapêutico necessário está sendo mantido; para isso, é utilizado o tempo de protrombina, cujo resultado pode ser expresso em segundos, em atividade de protrombina ou em INR, que devem ser realizados num intervalo de, no máximo, 4 semanas, como preconizado pelo American College of Chest Physicians. ${ }^{20}$

De acordo com alguns autores, de maneira geral, o intervalo terapêutico do INR deve ficar entre 2.0 e 3.5, mas, dependendo do tipo de doença apresentada pelo paciente, valores maiores de INR são considerados terapêuticos. ${ }^{3,6,8,21}$ Um valor de INR entre 2 e 3 é usual para controle de trombose venosa; já em pacientes portadores de válvulas cardíacas protéticas passa a ser necessário um INR por volta de 3.5.22

Em 1994, Lippert e Gutschik² publicaram recomendações onde o INR não deveria ser maior que 4.0, e preferencialmente menor que 3.0, antes do paciente anticoagulado ser submetido a procedimentos odontológicos com alto risco de sangramento.

As recomendações de alguns autores ${ }^{3,9}$ para vários procedimentos cirúrgicos odontológicos indicam que, para exodontias simples ou quando mínimo sangramento é esperado, um INR menor que 4.0 é aceitável; para casos de sangramento moderado, cirurgias de terceiros molares ou exodontias múltiplas, o INR deverá ser reduzido; em casos onde hemorragia maior é esperada, um INR menor que 3.0 é indicado; e, quando o INR estiver acima de 5.0, cirurgias não devem ser realizadas.

Evans et al. ${ }^{23}$ concluiram, em seu estudo, que pacientes usando dicumarínicos com INR menor que 4.1 podem ser submetidos a exodontias sem alteração na medicação. Já de acordo com Scully e Wolff, ${ }^{22}$ exodontias a fórceps não complicadas de um a três dentes, em pacientes com INR menor que 3.5 e sem outros fatores de risco (como uso de AAS, álcool, outras coagulopatias), associadas a hemostáticos locais podem ser realizadas sem alteração na medicação anticoagulante.

Dos casos avaliados nos prontuários, quatro exodontias foram de terceiros molares inclusos e uma caso de apicectomia, cirurgias estas que representam um traumatismo cirúrgico maior que uma exodontia simples, e nessas situações foi associado um hemostático local (Beriplast@) para auxiliar na prevenção de hemorragia, não tendo ocorrido sangramento no pós-operatório.

Vários autores preocuparam-se em salientar a necessidade do emprego da técnica cirúrgica atraumática e da aplicação de medidas locais convencionais de controle de hemostasia, onde a sutura adequada é extremamente importante $1,4,7,8,10,15,24-26$

A manutenção da medicação anticoagulante vem sendo preconizada por um número crescente de pesquisas, que observaram uma incidência mínima de episódios hemorrágicos após cirurgias onde os valores em TP e/ou em INR dos pacientes estavam dentro dos índices terapêuticos. ${ }^{1,4-8}$ Esse protocolo tem sido ainda mais reforçado por autores ${ }^{10-19,27}$ que enfatizam a utilização de hemostáticos locais, afirmando a eficiência dos mesmos na prevenção e controle de hemorragias pós-operatórias. Nosso levantamento mostrou resultados semelhantes com índice mínimo de sangramento pós-operatório (um caso) controlado por medidas locais com tamponamento.

Essas orientações também são preconizadas pelo Comitê Britânico de Hematologia, o qual descreve que o risco de sangramento nesses pacientes mantendo o INR entre 2 e 4 é pequeno para cirurgias odontológicas, sendo que a interrupção do anticoagulante não seria justificada devido ao aumento do risco de trombose. ${ }^{28}$

Dentre os materiais com ação hemostática citados na literatura, os mais utilizados são a esponja de gelatina reabsorvível, a esponja de colágeno, a celulose oxidada, o bochecho com ácido tranexâmico e o selante de fibrina. As pesquisas mostram que todos eles apresentam bons resultados na hemostasia local.

Autores relatam que o selante de fibrina apresentou ótimos resultados na prevenção de hemorragia e parece ser o hemostático com menos efeitos colaterais. . $3,25,26,29,30^{2}$

Assim, salientamos que a execução do procedimento cirúrgico com o menor trauma possível, a rigorosa observação de todos os passos da técnica cirúrgica, incluindo sutura adequada, e o seguimento das recomendações pós-operatórias pelo paciente devem ser fatores primordiais a serem considerados sempre em todos os pacientes e, especialmente, naqueles que se encontram anticoagulados.

A avaliação dos riscos de hemorragia trans e pósoperatórias, bem como da quantidade de trauma cirúrgico ao qual estará sujeito esse paciente, é imprescindível para o estabelecimento de um protocolo de atendimento adequado. O Comitê Britânico ressalta ainda a importância da habilidade e experiência do cirurgião-dentista. ${ }^{28}$

\section{Conclusão}

O estudo permitiu concluir que cirurgias odontológicas de pequeno e médio trauma podem ser realizadas com segurança mantendo-se a medicação em pacientes anticoagulados por dicumarínicos e com INR menor que 4 , desde 
que os princípios de técnica cirúrgica atraumática sejam seguidos rigorosamente e, se necessário, com o auxílio dos hemostáticos locais.

\begin{abstract}
In the daily medical practice there has been an increase in the use of anticoagulant drugs; as a consequence, dental offices are receiving more individuals under this therapy. Nowadays dental surgeons need a broader knowledge of hemostasis and associated therapies in order to offer the best possible assistance. This article presents a retrospective study based on the clinical records of anticoagulated patients using dicoumarin who underwent dental surgical procedures. Twenty-six patients were submitted to fortyseven minor dental surgical procedures without any change to their doses of anticoagulants. Laboratorial evaluations of PT (prothrombin time) based on the INR (International Normalized Ratio) were performed for all patients. The results showed that only one patient reported postoperative bleeding which was controlled by local compression. Based on the results of this study and a brief review of publications, the authors suggest that the most adequate conduct for most patients is to maintain anticoagulation therapy and the complementary use of local hemostasis when necessary. Rev. Bras. Hematol. Hemoter.
\end{abstract}

Key words: Anticoagulant therapy; oral surgery; local hemostatic.

\section{Referências Bibliográficas}

1. Bailey BM, Fordyce AM. Complications of dental extractions in patients receiving warfarin anticoagulant therapy. A controlled clinical trial. Br Dent J. 1983;155(9):308-10.

2. Lippert S, Gutschik E. Views of cardiac-valve prosthesis patients and their dentists on anticoagulation therapy. Scand J Dent Res. 1994;102(3):168-71.

3. Kwon PH, Laskin DM. Complications of dentoalveolar surgery. In: Clinician's manual of oral and maxillofacial surgery. 2. ed. 1997: 272.

4. Devani P, Lavery KM, Howell CJ. Dental extractions in patients on warfarin: is alteration of anticoagulant regime necessary? Br J Oral Maxillofac Surg. 1998;36(2):107-11.

5. Campbell JH, Alvarado F, Murray RA. Anticoagulation and minor oral surgery: should the anticoagulation regimen be altered? J Oral Maxillofac Surg. 2000;58(2):131-5.

6. Wahl MJ. Myths of dental surgery in patients receiving anticoagulant therapy. J Am Dent Assoc. 2000;131(1):77-81.

7. Dantas, A.K. Aspectos principais da propedêutica clínico-cirúrgica para pacientes em uso de anticoagulantes orais. Tese (Mestrado em Cirurgia e Traumatologia Buco-Maxilo-Faciais) - Faculdade de Odontologia da Universidade de São Paulo, São Paulo. 2002.

8. Gallardo FR. Atención odontológica de pacientes bajo tratamiento con anticoagulantes. Rev Fac Odontol Univ Chile 2001;19(2):54-60.

9. Jeske AH, Suchko GD. Lack of a scientific basis for routine discontinuation of oral anticoagulation therapy before dental treatment. J Am Dent Assoc. 2003;134(11):1492-7.

10. Martinowitz U, Mazar AL, Taicher S, Varon D, Gitel SN, Ramot B, et al. Dental extraction for patients on oral anticoagulant therapy. Oral Surg Oral Med Oral Pathol. 1990;70(3):274-7.

11. Souto JC, Oliver A, Zuazu-Jausoro I, Vives A, Fontcuberta J. Oral surgery in anticoagulated patients without reducing the dose of oral anticoagulant: a prospective randomized study. J Oral Maxillofac Surg. 1996;54(1):27-32.

12. Rada RE, Lamberghini F. Treatment of the patient receiving Coumadin anticoagulant therapy. CDS Rev. 1997;90(6):34-8.

13. Bodner L, Weinstein JM, Baumgarten AK. Efficacy of fibrin sealant in patients on various levels of oral anticoagulant undergoing oral surgery. Oral Surg Oral Med Oral Pathol Oral Radiol Endod. 1998;86(4):421-4.

14. Blinder D, Manor Y, Martinowitz U, Taicher S, Hashomer T. Dental extractions in patients maintained on continued oral anticoagulant: comparison of local hemostatic modalities. Oral Surg Oral Med Oral Pathol Oral Radiol Endod. 1999;88(2):137-40.

15. Halfpenny W, Fraser JS, Adlam DM. Comparison of 2 hemostatic agents for the prevention of postextraction hemorrhage in patients on anticoagulants. Oral Surg Oral Med Oral Pathol Oral Radiol Endod. 2001;92(3):257-9.

16. Cannon PD, Dharmar VT. Minor oral surgical procedures in patients on oral anticoagulants - a controlled study. Aust Dent J. 2003;48 (2):115-8.

17. Carter G, Goss AN, Lloyd J, Tocchetti R. Current concepts of the management of dental extractions for patients taking warfarin. Aust Dent J. 2003;48(2):89-96.

18. Davies D. Minor oral surgical procedures in patients on oral anticoagulants. Aust Dent J. 2003;48(4):267.

19. Beirne OR. Evidence to continue oral anticoagulant therapy for ambulatory oral surgery. J Oral Maxillofac Surg. 2005;63(4):540-5.

20. Hirsh J, Guyatt G, Albers GW, Harrington R, Schünemann HJ; American College of Chest Physicians. Executive summary: American College of Chest Physicians Evidence-Based Clinical Practice Guidelines (8th Edition). Chest. 2008;133(6 Suppl):71S-109S.

21. Aldous JA, Olson CJ. Managing patients on warfarin therapy: a case report. Spec Care Dentist. 2001;21(3):109-12.

22. Scully C, Wolff A. Oral surgery in patients on anticoagulant therapy. Oral Surg Oral Med Oral Pathol Oral Radiol Endod. 2002;94(1):57-64.

23. Evans IL, Sayers MS, Gibbons AJ, Price G, Snooks H, Sugar AW. Can warfarin be continued during dental extraction? Results of a randomized controlled trial. Br J Oral Maxillofac Surg. 2002;40 (3):248-52.

24. Carr MM, Mason RB. Dental management of anticoagulated patients. J Can Dent Assoc. 1992;58(10):838-44.

25. Dunn CJ, Goa KL. Fibrin sealant: a review of its use in surgery and endoscopy. Drugs. 1999;58(5):863-86.

26. Spotnitz WD. Commercial fibrin sealants in surgical care. Am J Surg. 2001;182(2 Suppl):8S-14S

27. Schardt-Sacco D. Update on coagulopathies. Oral Surg Oral Med Oral Pathol Oral Radiol Endod. 2000;90(5):559-63.

28. British Committee for Standards in Haematology. Guidelines for the management of patients on oral anticoagulants requiring dental surgery. www.bcshguidelines.com

29. Okamoto T, Alves-Rezende MC, Okamoto AC, Buscariolo IA, Garcia IR Jr. Osseous regeneration in the presence of fibrin adhesive material (Tissucol) and epsilon-aminocaproic acid (EACA). Braz Dent J. 1995;6(2):77-83.

30. Romanos GE, Strub JR. Effect of Tissucol on connective tissue matrix during wound healing: an immunohistochemical study in rat skin. J Biomed Mater Res. 1998;39(3):462-8

Avaliação: Editor e dois revisores externos

Conflito de interesse: sem conflito de interesse

Recebido: 28/11/2008

Aceito após modificações: 13/04/2009 